\title{
Study on the Willingness of Hospital $J$ to Participate in Station-based Assistance in Medical Alliance
}

\author{
Cancheng Zhang, Mengying Deng* \\ The First Affiliated Hospital of Jinan University, Guangzhou, China \\ Email address: \\ zhangcc@jnu.edu.cn (Cancheng Zhang),dmy114@jnu.edu.cn (Mengying Deng) \\ ${ }^{*}$ Corresponding author
}

\section{To cite this article:}

Cancheng Zhang, Mengying Deng. Study on the Willingness of Hospital J to Participate in Station-based Assistance in Medical Alliance. World Journal of Public Health. Vol. 5, No. 2, 2020, pp. 37-41. doi: 10.11648/j.wjph.20200502.12

Received: April 28, 2020; Accepted: May 18, 2020; Published: May 28, 2020

\begin{abstract}
Medical assistance is an important way to address the uneven distribution of medical resources and promote health equity. The medical alliance is the practice of medical administrative authorities and hospitals to explore the development of medical assistance, but at present there are difficulties in this cooperation. In this study, we analyze the practice of Hospital $\mathrm{J}$ in helping member hospitals in the construction of a medical alliance, and the key influencing factors of cooperation at three levels: hospital leaders, department heads and general practitioners. We propose a research design, focusing on how to enhance the level of willingness of core hospitals to participate in the cooperation of station-based support, which is useful for enhancing the leading role of core hospitals, promoting the steady development and achieving the policy objectives of the medical alliance.
\end{abstract}

Keywords: Medical Alliance, Core Hospitals, Station-based Assistance, Willingness of Collaboration

\section{Introduction}

In recent years, China has been paying more and more attention to the development of medical assistance and exploring the model of medical alliance. Through the alliance, the help from big cities' tertiary hospitals to primary hospitals in small towns nearby and the implementation of graded diagnosis and treatment policy can be an effective way to solve the "difficult to see a doctor" problem in China.

In 2009, the State Council of the People's Republic of China proposed and encouraged the exploration of the institutional mechanism of the medical alliance to drive the improvement of the capacity of primary health care services. [1] In September 2015, the General Office of the State Council issued Guiding Opinions on Promoting the Construction of a Graded Diagnostic and Treatment System, which formally put forward the goal of "focusing on the strong grassroots level and improving the construction of a graded diagnosis and treatment model". [2] In August 2016, the National Health and Family Planning Commission (later renamed National Health Commission) stated in Notice on the Promotion of Pilot Work on Hierarchical Treatment that the medical alliance is an important component of hierarchical treatment. On March 5, 2017, Report on the Work of the Government emphasized that all tertiary public hospitals should participate and play a leading role.

In order to effectively achieve the goal of medical assistance, some parts of China have explored different forms of medical alliance models. For example, in Shanghai, there are models such as " $3+2+1 ", 3+" 2+1 "$ and " $3+2 "+1$ [3]; in Hubei Province, there are six types of alliance models: medical group model, hospital hosting model, medical collaboration model, telemedicine model, direct management community model and private hosting model [4]; the city of Dalian is committed to the development of a regional medical alliance based on telemedicine systems. [5]

At present, various regions and hospitals in Guangdong Province are providing medical assistance in various ways, gradually forming various forms of medical alliance, including the formation of medical groups in cities, medical communities in counties, specialist alliances across regions and telemedicine collaboration networks in remote and poor areas. The connotation of the medical alliance emphasizes cooperation between different levels of medical institutions, especially between large urban hospitals and primary 
hospitals. [6] The aim of medical assistance is to achieve interregional cooperation and resource integration, in line with China's current situation. [7] The public interest can only be fully realized if public hospitals assume responsibility for improving the health of the population, which is also a requirement for their sustainable development. [8] However, in some major cities, hospitals are not doing enough to help lower-level hospitals, and their motivation is worrying. With the implementation of medical assistance in the medical alliance, the interest pattern of core hospitals, departments and doctors will change, and the core hospitals' willingness to maintain the cooperation becomes difficult in the long term.

There has been a great deal of fruitful research on the medical alliance over the past 30 years, but less specific research on the willingness of core hospitals to cooperate, especially when it comes to specific support tools such as station-based assistance. In this paper, we analyze the current situation of Hospital J's participation in the medical alliance, and dig into the key factors affecting the willingness of hospital leaders, department heads and general practitioners to cooperate, so as to summarize the problems in the process of medical alliance cooperation, and try to put forward suggestions of solving the problems, hoping the policy objectives of the medical alliance will be realized in the near future.

\section{Status of Hospital J's Participation in the Construction of the Medical Alliance}

Hospital J was officially established in 1981 in Guangzhou. It is an affiliated hospital of University $\mathrm{J}$, a key university of the "211 Project", and is a comprehensive tertiary A hospital. The hospital has been exploring and practicing external medical cooperation, but in a relatively fragmented and unsystematic way. The construction of a true medical alliance was officially conducted in October 2015.

As a core hospital, Hospital $\mathrm{J}$ built the Tianhe District Medical Alliance in October 2015. In terms of type, Tianhe District Medical Alliance is a loose medical alliance. In this model, there is a lack of common goals of interest among group members, weak cooperative relationships, and less stable cooperation. In Hospital $\mathrm{J}$, the willingness of the doctors assigned by their superiors to participate in the station-based support in the Alliance is at an average level.

In December 2017, Hospital J carried out a comprehensive co-construction and trusteeship in Hospital L in Longmen, Huizhou, making full use of its medical resources as a tertiary A hospital, helping Longmen County improve its medical and health service capacity, assisting in the construction of the new site of Hospital $\mathrm{L}$ and promoting the construction and development of the medical alliance. Hospital J has so far sent out 6 groups, 42 people in total, to participate in the assistance. Longmen Alliance is a model of so-called semi-compact alliance, which is based on co- hosting of both sides. In this model, both parties can practice a strong cooperative relationship. In general, Hospital L hopes that the medical assistance of Hospital $\mathrm{J}$ will maintain systemic and continuous, and that medical experts from Hospital J can stay for a longer time, guiding the doctors and staffs in Hospital L. Due to the imperfect incentive mechanism, the geographical distance of Longmen County from Guangzhou, as well as the poor medical base and so on, the willingness of some departments and general practitioners in Hospital $\mathbf{J}$ to cooperate is at an average or low level.

In summary, Hospital $\mathrm{J}$, as a core hospital participating in the construction of the medical alliance, plays a leading role. From a praxeological point of view, the key element in the generation of behavior is willingness. The willingness of public organizations to cooperate is shaped by a combination of internal and external, managerial and environmental factors that first affect the organization's level of resource self-sufficiency, the degree of external facilitation of cooperation and the organization's cultural openness, and then ultimately its willingness to cooperate. [9] The high or low willingness of hospital leaders, department heads and general practitioners to cooperate determines the level of support and motivation, which ultimately affects the effectiveness of the medical alliance.

\section{Survey on Hospital J's Willingness to Participate in the Medical Alliance}

This paper uses a case study approach, based on Hospital J's participation in the medical alliance. We analyze the key influencing factors of collaboration for three levels: the hospital leadership, department heads and general practitioners. The interview method was used for hospital leaders and department heads; while for general practitioners, we conducted questionnaires due to the large number of people. The results of the survey show that the willingness to cooperate and the problems at the three levels are different, and on this basis, we propose some measures and suggestions to strengthen the willingness of the core hospitals to participate in the cooperation and station-based assistance.

According to the stakeholder theory, the construction of a medical alliance should take the integration of interests as the entry point to coordinate and deal with the interests among hospitals and medical personnel. [6] It was found from the survey that the stakeholders at different levels of Hospital J (hospital leaders, department heads and general practitioners) had different perceptions and understanding of the stationbased assistance. Among them, general practitioners ' perceptions are generally better. However, the hospital leadership has a high willingness to cooperate, followed by the department heads and general practitioners. We also found that the key influencers of concern vary across different levels of respondents.

Technical assistance can improve the professional quality and management level of doctors in the station hospital, but 
since this method requires not only the dedication of the station hospital, but also policy support and financial investment, continuity is difficult to maintain. [10] Hospital leaders believe that there are two main factors that affect the willingness to cooperate: (1) the cooperation is not strong enough to form a community of interest; (2) The government support is not strong enough, and the policy support is difficult to match.

The department heads generally believe that there are three factors affecting the collaboration: (1) the shortage of staff in the department due to outstation help; (2) The lack of a mechanism for division of labor and collaboration, affecting the enthusiasm of the department to participate in the assistance; (3) "pulling and matching" in the alliance is the norm, which means the department does not have enough autonomy to choose.

Through the questionnaires and previous interviews with the general practitioners, it can be found that the demographic characteristics of doctors, such as gender, age, marital status and family situation, have a certain influence on their willingness to participate in the alliance, and have a weakening or strengthening effect on the likelihood of participation and the degree of effort in helping the doctors in the station hospital. General practitioners' willingness to cooperate is at an average or low level, and various negative factors make them apprehensive about cooperating.

\section{Analysis of the Reasons for the Low Willingness of General Practitioners to Cooperate}

Whether or not they have participated in the station-based support in the medical alliance, doctors in Hospital $\mathrm{J}$ have similar perceptions on the current lack of medical assistance. From the results of the questionnaire combined with the previous interviews, the reasons for the lack of willingness of doctors to cooperate can be summarized as the following.

\subsection{Performance Evaluation Criteria are Not Developed to Properly Assess the Effectiveness of Assistance}

Neither the core hospital nor the station hospital has formed a set of effective performance evaluation criteria to quantify the work performance of doctors in the station hospital. In particular, "evaluation criteria" means the value scale for evaluating a specific doctor. It is important that the criteria be objective.

Based on the fact that the work of physicians and surgeons varies considerably in terms of the content of their work and the need for assistance varies from one department to another, it is necessary to draw up a performance evaluation criteria sheet for each individual in order to be objective and fair. Combing through the performance evaluation criteria requires a considerable amount of work, a part of which is somewhat neglected.

\subsection{Inadequate Incentive Mechanisms and Failure to Reflect the Rights and Interests of Participating Doctors}

The participating doctors in the medical alliance mostly value the rewarding benefits, including merit-based compensation, promotion benefits, personal branding, therapeutic autonomy and academic pursuits.

We found from the survey that the current incentive mechanism of the alliance to help the cooperation is not satisfactory to the doctors. The cooperation agreement is only a framework agreement, and it is normal that, after the negotiation on the cooperation projects and goals, there are some small changes in practice. The doctors are mostly worried that the goals change at any time, the number of tasks increases at any time, and promised benefits never arrive.

The remuneration for the labor of experts stationed at Hospital $\mathrm{J}$ has not been included in the performance appraisal system of the member hospitals of the Tianhe District Medical Alliance or Hospital L, but has been issued in a separate register as agreed by both parties, without forming a community of interest.

\subsection{Lack of Attention to Cultural Integration and Difficulty in Ensuring Long-term Stability of Cooperation}

For various reasons, it is relatively easy for hospital management within the medical alliance to reach consensus, but there is often a lack of cultural integration and cooperative consensus at the individual medical staff or departmental level, which affects the effectiveness of alliance cooperation.

\section{Responses to Increase Willingness to Cooperate in Core Hospitals}

\subsection{Clarify the Roles of All Parties and Establish a Mechanism for Sharing the Benefits of the Medical Alliance}

Classifying the stakeholders and proposing management strategies is conducive to the establishment of a long-lasting balance of interests to promote the effective operation of the medical alliance. [11] It is important to effectively deal with the interests of different parties, such that fairness, efficiency and safety in the medical alliance can be realized and guaranteed. In this way, general public's increasing demand for health services can truly be met, and patients can obtain cost-effective medical services. This is the ultimate mission of the medical alliance. [12]

The core hospitals should focus on the export of technology, management, branding and the deployment of specialist teams in the station-based assistance, amplifying the value of quality medical resources and continuously reproducing the values in core hospitals.

Member hospitals should improve their management and technical level with the help of core hospitals to enhance 
regional medical service capacity, and in the mean time enhancing social and economic benefits on the premise of public welfare.

The government and health authorities adjust their management methods according to the actual situation, strictly function in planning, regulation and supervision, and play a leading role in the practice of supporting the medical alliance.

The core stakeholders of the medical alliance must be clear about their roles and responsibilities. Only by ensuring the benefits of the core hospital will they have the incentive to participate in the medical assistance.

\subsection{Increase Policy Guidance and Strengthen Support}

From the interviews, it is learned that Hospital $\mathbf{J}$ expects more from the government's input and believes it should play a greater role. Therefore, the government should guide the participants in the framework of the medical alliance to cooperate, coordinate the development, optimize the allocation of resources, and recognize the leading role of the core hospitals.

It is difficult for a medical alliance to sustain if the core hospitals do not get management and financial support. Only through strong government policy guidance and resource support can the core hospitals be relieved of the worries of participating in the medical assistance.

\subsection{Construct a Reasonable Division of Labor and Cooperation Mechanism to Ensure the Stability of Assistance}

In order to enhance the willingness of the experts in the medical assistance to cooperate, and to work in a consistent and efficient manner with the member hospitals, the construction and implementation of a reasonable mechanism for the division of labor in the alliance is the guarantee and basis.

The division of benefits between the core hospital and the member hospitals for the treatment of specific diseases can be conducted by selecting the specific department as a unit, hence further improving and strengthening the mechanism of division of labor and collaboration. In addition to external economic benefits, the sharing of resources among medical institutions is an important means of increasing the willingness to cooperate.

\subsection{Increase the Number of Staff in the Units and Respect the Realities of the Units by Matching Support to Promote Free Love"}

After some preliminary discussions, hospital leaders of all parties in the medical alliance should give more autonomy to the departments, and fully respect the right of the departments to choose. The mode of cooperation should go from "pulling and matching" to "free love".

"Free love" means that the two hospitals in the alliance and the two departments have a certain degree of autonomy. One side is willing to help and the other is willing to accept the help. Both sides can maintain their independence, but also can be consistent, and ultimately achieve the rational allocation of medical resources.

\subsection{Improve Appraisal and Incentive Mechanisms with Due Regard to Doctors' Demands}

For the core stakeholders of the alliance, especially the core hospitals and their staffs, a management-focused strategy is recommended, in which their interests are fully analyzed and addressed as fully as possible, in order to promote their acceptance and support for the alliance. [13]

We found that a well-developed incentive mechanism is a crucial influencing factor for doctors in Hospital J. Hence the establishment of such a mechanism should be at the core of the development of a medical alliance.

We suggest that, in order to increase the enthusiasm of doctors to participate in the station-based assistance, the general performance appraisal should be refined under regulations, and performance appraisal rules should be set up to suit the characteristics and forms of each doctor's department.

\subsection{Focus on Cultural Integration to Enhance Value Identity}

The construction of a medical alliance is also a process of convergence of multiple cultures into a new culture of superiority, and the reorganization of resources between different medical institutions is the absorption of the superior culture into other relatively disadvantaged cultures. [14]

A harmonious interpersonal relationship is one of the important conditions for a harmonious working atmosphere. [15] The core hospitals should be more proactive in understanding the culture of the member hospitals, and only by achieving a deeper cultural integration can the construction of a medical alliance be realized, thus increasing the willingness of the core hospitals to participate in the medical alliance.

\section{References}

[1] State Council of the People's Republic of China. Opinions on deepening the reform of the medical and health system $[\mathrm{Z}]$.

[2] Siyi Luo, Jian Tan, Meifang Zhou. An empirical study on the perception and demand for medical liability insurance among medical personnel in Guangzhou [J]. China Health Insurance, 2015 (8): 54-56.

[3] Jieming Qu, Weiping Li, Bo Yan, et al. Exploring the reform of vertical integration of medical resources in Shanghai [J]. Chinese Journal of Hospital Management, 2011, 27 (7): 499502 .

[4] Yan Xiao, Xiaoming Ruan. The practice of medical consortia in Hubei region. The practice and thinking of the Hubei regional medical consortium [J]. China Hospital Management, 2012, 32 (10): 12-13. 
[5] Xizhuo Sun, Fangfang Gong, Xiaodong Gu, et al. The practice and exploration of remote regional medical consortium [J]. Modern Hospital Management, 2013, 11 (3): 8-10.

[6] Mengfei Li. A study on the status and countermeasures for the development of "medical alliance" in China [D]. Shandong University, 2017.

[7] Kaibin Zhong. Counterpart support: origin, formation and evolution. Journal of Gansu Administrative College, 2013 (04):pp. 14-24+125-126.

[8] Bin Li, Rongming Ren, and Hui Han. Social responsibility in public hospitals from a stakeholder perspective. Southwest National Defense Med, 2012 (04): 432-434.

[9] Conglin Fan. A study of factors influencing the willingness of public organizations to cooperate [J]. Business Times, 2013 (14): 93-95.

[10] Juanjuan Lin and Xiaochang Chen. Analysis of the key problems of building medical consortia and their countermeasures [J]. Journal of Nanjing Medical University (Social Sciences Edition), 2014 (2): 104-108.
[11] Xiyi Xia, et al. The practice and thinking of counterpart support in urban and rural hospitals. Jiangsu Health Business Management, 2009 (02): 35-36.

[12] Yihua Xu, Chun Chen, Tao Wang, Mengtao Zhou. Advantages and disadvantages of regional medical consortia from a stakeholder perspective [J]. Medicine and Philosophy (B), 2015, 36 (12): 79-81.

[13] Nian Zhang, Changying Yu, Lingli Chen, Shifang Liu, Huihua Huang. A study on the definition and classification of stakeholders in medical alliance [J]. Anhui Medicine, 2017, 21 (09): 1747-1750.

[14] Shimei Jiang, Xiu Zhong, Sheng Zhong. An exploration of cultural integration in the construction of regional medical alliance [J]. Chinese Medical Ethics, 2016, 29 (2): 327-329.

[15] Shuguang Li, Yuhong Jiang, Yanhong Wu. Building good interpersonal relationships in hospitals [J]. Chinese Journal of Hospital Management, 2001, 17 (5): 303-304. 